\title{
MAGNETIC FLUIDS AND THEIR APPLICATIONS
}

\begin{abstract}
Magnetic fluids contain magnetic nanoparticles which are dispersed in water or various types of oils. Magnetic nanoparticles are monodomain and their magnetic moments are oriented in the direction of the magnetic field. They interact with each other and create new structures, which depend on parameters of magnetic nanoparticles, temperature, values, and development of magnetic and electric fields. This paper describes two basic methods of investigation of magnetic fluids in magnetic and electric fields by the acoustic and the dielectric spectroscopy. Magnetic fluids have wide application in technology, medicine and other areas.
\end{abstract}

Keywords: magnetic nanoparticles, dielectric, acoustic, spectroscopy

\section{Introduction}

Nano-science deals with a study of objects, chains or clusters of atoms and molecules. At least one of their dimension is in the range of one to several tithes of nanometers $\left(1 \mathrm{~nm}=10^{-9} \mathrm{~m}\right)$. NANO (Figure 1). An object of such size compared to a human is about as small as human compared to the Sun. Even a few decades ago, the idea of small things was often identified with micro-objects - the "micro" was a trend. We had microscopes, micro-organisms, microphones, microwaves, microprocessors. The technologies are limited by imaging and manufacturing techniques, which allowed only the structures with the smallest dimensions to be several microns $\left(10^{-6} \mathrm{~m}\right)[1]$.

Today it is known that atoms are formed from protons, neutrons and other elementary particles like electrons or quarks. Their numbers and distribution in given atom (element) determine its parameters and properties. Each known elements as oxygen, carbon, gold or others are placed according to the properties of their atoms in the periodic table. At present, more than 100 different atoms are known. Many of them naturally occur in the universe, but some have been created artificially. The dimensions of each atom are several angstroms, respectively, several tenths of a nanometer $\left(1 \AA=0.1 \mathrm{~nm}=10^{-10} \mathrm{~m}\right)$. The space between atoms, nano-objects and micro-objects is filled by a nano-world a world of very special, small structures with various shapes and marvelous properties. However, all these phenomena and objects are invisible to us, although some of their properties can be registered and used in a macro-world.

\section{Magnetic nanoparticles}

Nano-objects exist in different shapes, forms and size. They can be nano-particles, nano-fibers and other various nanostructured objects, or they can be parts of other materials with which they create nano-composite materials offering new possibilities and combinations of properties. From the many shapes and compositions of nano-objects we will focus only on a special group of magnetic nanoparticles with a size between 5-30 $\mathrm{nm}$, which consist of iron oxides with good magnetic properties and also high oxidation resistance. A magnetic fluid (MF) consists of the magnetic nanoparticles coated with a surfactant, which are dispersed in carrier fluid based on water or oil. From the chemical point of view, the carrier fluid must be stable for the required temperature range.

MF is formed by magnetic nanoparticles (usually magnetite, $\mathrm{Fe}_{3} \mathrm{O}_{4}$ ) coated with surfactant agents (amphiphilic molecules, as oleic acid and aerosol sodium di-2 ethylhexyl-sulfosuccinate) in order to prevent their aggregation in a carrier fluid. Stefic repulsion among nanoparticles acts as a physical barrier [2] that keeps nanoparticles in a solution and stabilizes a MF. If the nanoparticles are dispersed in a nonpolar medium, such as oil, only one layer of surfactant is needed to form an external hydrophobic layer (Figure 2a). The polar head of a surfactant is attached to the nanoparticles surface, and the carbonic chain is in contact with the carrier fluid. On the other hand, if the particles are dispersed in a polar medium, such as water, a double surfactation of the particles is needed to form a hydrophilic layer

\footnotetext{
* ${ }^{1}$ Jozef Kudelcik, ${ }^{2}$ Maria Kudelcikova

${ }^{1}$ Department of Physics, Faculty of Electrical Engineering, University of Zilina, Slovakia,

${ }^{2}$ Department of Structural Mechanics and Applied Mathematics, Faculty of Civil Engineering, University of Zilina, Slovakia

Email: jozef.kudelcik@fel.uniza.sk
} 


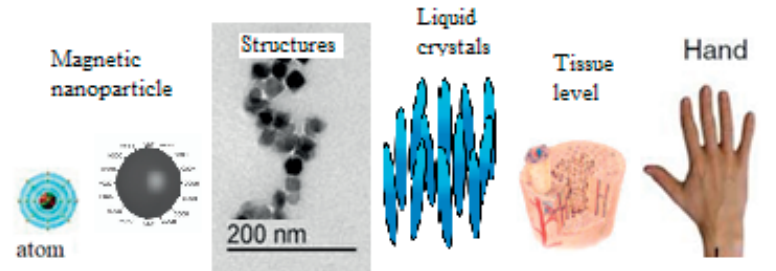

$\begin{array}{llllllllll}0.1 \mathrm{~nm} & 1 & 10 & 100 & 1 \mu \mathrm{m} & 10 & 100 & 1 \mathrm{~mm} & 10 & 100\end{array}$

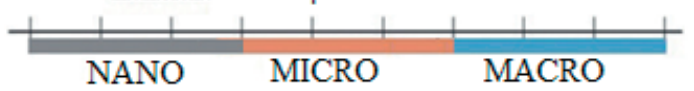

Figure 1 Nano-objects, micro-objects and macro-objects

around them (Figure $2 b$ ). The polar heads of surfactant molecules can be cationic, anionic or nonionic [3].

Although magnetic nanoparticles are objects from nano-world, we do not need Quantum mechanics to describe their motion in space. The motion of a magnetic nanoparticle in a carrier fluid can be described using terms from Classical mechanics, and it is governed by several factors including (a) the magnetic force, (b) viscous drag, (c) particle-fluid interactions (perturbations to the flow field), (e) gravity/buoyancy, (g) thermal kinetics (Brownian motion), and (h) interparticle effects including (1) magneticdipole interactions, (2) electric double-layer interactions, and (3) van der Waals force.

For the basic analysis, we consider nanoparticles in low concentration, and we neglect particle/fluid interactions and interparticle effects. In many applications, particle diffusion due to Brownian motion can also be neglected, what simplifies the analysis. However, for a correct understanding of given application, Brownian motion cannot be ignored, as this motion can have various effects in the region of interest. Next important information is that a real MF does not contain magnetic nanoparticles with a single size but contains a range of different particles size. In order to take into account the effects of polydispersity, the lognormal distribution function is usually used in theoretical predictions [5], [6]. The typical size of the magnetic nanoparticles in a MF is of the order of $10 \mathrm{~nm}$, sufficiently small for them to be magnetically monodomain. This is an important characteristic; because the particles must have non-zero magnetic moments for the MF to show its paramagnetic properties. The main property of the MF is that, in the presence of a nonhomogeneous magnetic field, particles are rotated and attracted to the region where the field intensity is maximal.

Two distinct mechanisms exist for the rotation of the magnetic moments in magnetic fluids [6]. One of them is the rotation of the whole magnetic particle inside the fluid carrier, known by the name of Brownian rotation (in the absence of a magnetic field, the particle rotates due to the Brownian torques (molecular collisions)). The relaxation time for spherical nanoparticles is dependent on its volume and the fluid's viscosity.
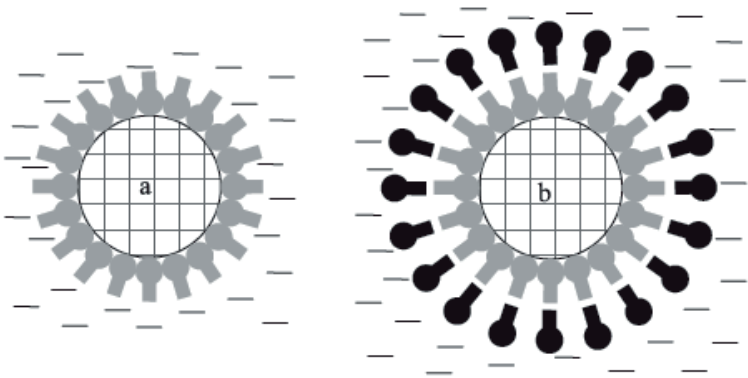

Figure 2 A nanoparticle in a non-polar solvent (a) and in a polar solvent (b) [4]

The other mechanism is the rotation of the magnetic moment with respect to the nanoparticle, known as the Neel rotation. The relaxation time for this rotation is strongly dependent on the nanoparticle's volume and the temperature. When the Neel rotation is the dominant mechanism (temperatures higher than blocking temperature $-T_{B}$ ), i.e., when the magnetic moment is quasi-free to rotate, the nanoparticle is superparamagnetic. Bellow $T_{B}$ the magnetic fluid is still superparamagnetic because the magnetic nanoparticle, and hence also its moments, continues to be quasi-free to rotate. When nanoparticles are exposed to an $\mathrm{AC}$ magnetic field with the period less than their magnetic relaxation times of nanoparticles, heat is dissipated due to the delay of the relaxation process to the magnetic moment.

At present three main theoretical models and some simulations are used to describe the arrangement of magnetic nanoparticles in a magnetic field [7], [8], [9], [10], [11], [12]. It is also well known that under the effect of a magnetic field the nanoparticles in a magnetic fluid arrange into chains or structures with various shapes along the magnetic field direction. The parameters of these structures can be determined by comparison of theoretical predictions with measured results of the anisotropy of acoustic attenuation in the magnetic field. The first theoretical description of the anisotropy of acoustic attenuation of a magnetic fluid under the external magnetic field was published in 1986 by Taketomi [7]. He assumed that magnetic nanoparticles form the spherical clusters of some radius $a$, which create long chains, aligned in magnetic field direction (Figure 3, $\mathrm{h} \sim 1$ ). The idea that magnetic nanoparticles in the presence of the external magnetic field create only chains and they do not form clusters was presented in work by Pleiner and Brand [13]. Based on this assumption Shliomis et all [9] prepared theoretical model which takes into account only oligomers: dimers, trimers or short thin chains (Figure $3, \mathrm{~h} \gg>1$ ). Next interpretation of acoustic attenuation in magnetic fluids is based on the theoretical model proposed by Ahuja and Hendee [8]. In their theory, it is assumed that structures of magnetic nanoparticles in the magnetic field have various forms as oblate or prolate spheroidals, spherical, disk-like and needle-like shapes (Figure 3, $\mathrm{h}>1$ ). 


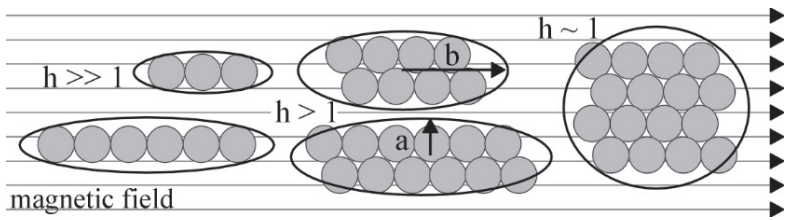

Figure 3 Types of possible ellipsoid aggregates that can be formed in the magnetic fluid under the effect of magnetic field: chain-like $(h=b / a \gg 1)$, prolate spheroid $(h>1)$ and sphere $(h \sim 1)$

\section{Application of magnetic fluids}

Magnetic fluids or ferrofluids are smart fluids [14], [15], [16], [17], which are rather attractive for a large variety of applications, which require fluid and magnetic properties simultaneously. Macroscopically, these fluids manifest themselves as magnetizable fluid media due to the "integration" of nanosized permanent magnetic nanoparticles in the carrier structure. The main feature of the research area in magnetic fluids or ferrofluids is high applicability of these materials. During the last century, chemists and physicists made a big effort to synthesize stable magnetic fluids, motivated by the perspective of many important technological uses. A clear confirmation of these perspectives was that from 1970 the number of patents is about half the numbers of published papers each year [3].

The research field of magnetic fluids is a multi-disciplinary area: Chemists study their synthesis and produce the ferrofluids, physicists study their physical properties and propose theories which explain them, engineers study their applicability and use them in technological products, biologists and physicians study their biomedical possibilities and use them in medicine. Most applications of magnetic fluid are based on its following properties [3], [12], [18]:

1) It goes to where the magnetic field is the strongest and stays there;

2) It absorbs electromagnetic energy at convenient frequencies and heats up;

3) Its physical properties may change with the application of a magnetic field.

These properties make MFs useful for many technological, biological and medical purposes, and they can also help in materials science and engineering research. From technological applications of MFs we describe these four main categories: a) Dynamic sealing; b) Heat dissipation; c) Damping; d) Doping of technological materials [3], [18]. One of many applications of heat dissipation using MF is a loudspeaker, whose coil heats up by functioning. In this case, MF is kept in place by the magnetic field of the magnet which is fixed on the loudspeaker's horn. The presence of MF around the coil also improves the quality of the speaker because unwanted resonances are damped. Next more direct use of MF for damping of unwanted vibrations is associated with their use as inertial and viscous dampers for motors, mainly stepper motors.
From the several possibilities of use of ferrofluids as technological materials, we have chosen only the case of liquid crystals doping, which is studied also in our laboratory. The doping of liquid crystals by magnetic nanoparticles reduces the magnetic field necessary to orient the liquid molecules more than hundred times [19], [20]. According to experimental observations with lyotropic nematic liquid crystals [21], above a minimum value of the concentration of particles, the liquid crystalline matrix collectively follows the orientation of the magnetic particles. Magnetic nanoparticles can also be used to investigate dynamic processes in lyotropic ferronematics (nematic liquid crystals doped with ferrofluids), in particular, the response of the nematic matrix to magnetic fields [22]. The next area of study is an investigation of their elastic properties. The bend elastic constant and the anisotropy of the diamagnetic susceptibility can be measured by comparing the relaxation behavior of liquid crystals with and without the ferrofluid doping in different magnetic fields. Magnetic nanoparticles significantly increase the sample light absorption, which is particularly useful to investigate the nonlinear [23] optical properties of lyotropic liquid crystals. The nonlinear refraction index of doped samples was shown to be ten times larger than those of undoped samples [24].

The main categories in biomedical applications are a) Magnetic drug targeting; b) Hyperthermia; c) Contrast enhancement for Magnetic Resonance Imaging - MRI; d) Magnetic separation of cells [3]. Localizability of MF by a magnetic field offers very interesting applicability in medicine, because any fluid may be turned into a magnetic fluid. Much attention has been paid to bounding on ferrofluids chemical drugs appropriate for chemotherapy. The idea is simple: such a ferrofluid bounded drug is injected into a cancer tumor, and there it is kept during some time (one hour) by a suitably focused magnetic field. The amount of drug necessary is much less than what would be necessary if it were dispersed in the whole body. When the magnetic field is turned off the drug will disperse in the body, but, since the total amount was very small, there will be practically no side effects. The hyperthermia is based on the absorption property of ferrofluids because they can absorb electromagnetic energy at the frequency that is different from the frequency at which water absorbs energy. This allows one to heat up a localized portion of a living body, where ferrofluid has been injected, for example, a tumor, without heating at the same time the surrounding parts of the body. MRI has been one of the most powerful diagnosis techniques used in medicine in recent years. Its ability to distinguish between different tissues relies on the different relaxation times of the proton's magnetic moments when it is in different ambient. Magnetic particles from a biocompatible ferrofluid are selectively absorbed by the kind of tissue, so they them very clearly visible by MRI. Moreover, different tissues uptake different amounts of the magnetic particles, so distinguishable images have different values. 


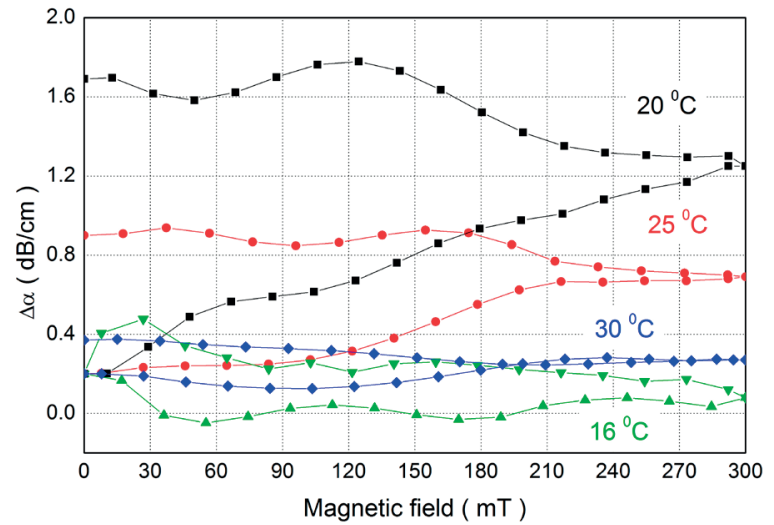

Figure 4 The temperature influence of the attenuation changes on the external magnetic field for $1.5 \%$ MF based on transformer oil MIDEL ( $15{ }^{\circ} \mathrm{C}, \boldsymbol{\Delta} 25^{\circ} \mathrm{C}, \overrightarrow{30}{ }^{\circ} \mathrm{C}$ )

\section{Experimental part}

The block diagrams of the experimental arrangements are shown in works [25], [26]. In the case of measurements of acoustic properties, MF was placed in the measuring cell of volume around $2 \mathrm{~mL}$. The measurements of the acoustic attenuation at the frequency $13 \mathrm{MHz}$ were carried out by a pulse method using the Workstation EXPLORER II. An acoustic wave is generated by transducers located in measuring cell, propagated through the MF, and underwent multiple reflections between transducers. The acoustic signal is received by the second transducer, registered by Workstation and displayed by the oscilloscope. At measurements of the dielectric properties of MF we used commercial cells for liquid crystal (LC) as capacitors. The cells are composed of two transparent flat glass (sandwiched type) substrates coated with Indium Tin Oxide (ITO) conductive layers acting as electrodes. The distance of two parallel plate ITO electrodes was $d=5 \mu \mathrm{m}$ and the active electrode area was $A=30 \mathrm{~mm}^{2}$. The capacitance of the air filled cell was $C_{0}=56 \mathrm{pF}$. At both types of measurements cells were placed on thermally stabilized blocks, the temperature of which was stabilized by the thermostat in the range from $10^{\circ} \mathrm{C}$ to $55^{\circ} \mathrm{C}$ with accuracy $\pm 0.1{ }^{\circ} \mathrm{C}$. During the measurement, the cells were inserted in the electromagnet, where the magnetic field was controlled by the current source. The whole measurement was controlled by the computer.

The acoustic attenuation was investigated for various types or concentrations of MFs [25], [26], [27]. These results showed a strong influence of a magnetic field on the value of acoustic attenuation. When the external magnetic field is increased, the interactions between the external magnetic field and the magnetic moment of the particle lead to aggregation of particles and various structures as chains or clusters are formed. These structural changes caused an increase in the acoustic attenuation. The decrease of the acoustic attenuation was slower at decreasing magnetic field so that a hysteresis effect can be observed (Figure

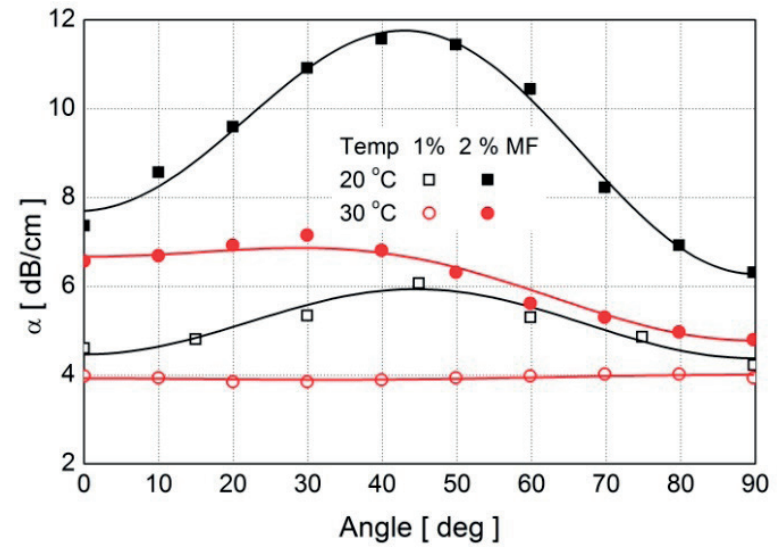

Figure 5 The anisotropy measurement of the acoustic attenuation for 1 and $2 \%$ magnetic fluid based on TECHNOL in the external magnetic field $(B=200 \mathrm{mT})$ for frequency $f=12.6 \mathrm{MHz}$ measured at two temperatures

4). This effect can be described by the existence of structures, the lifetimes of which were longer than the time of decrease of the magnetic field. Created structures do not disintegrate as soon as the magnetic field is removed.

A change of the acoustic attenuation with a magnetic field is also dependent on the temperature [6], [26], [28]. Its development for the magnetic fluid based on transformer oil MIDEL in the temperature range of $16-30{ }^{\circ} \mathrm{C}$ is presented in Figure 4. The measurements of the acoustic attenuation at temperatures $20{ }^{\circ} \mathrm{C}$ and $25{ }^{\circ} \mathrm{C}$ show the largest changes originated from the process of structures creation and show also higher hysteresis. At these temperatures, the creation of structures is more effective because the Brownian thermal motion has not energy sufficient to destroy them. At a higher temperature collision caused by thermal motion are more often and effectively. These collisions cause a decrease in numbers of clusters and their lengths with higher probability. Above $30{ }^{\circ} \mathrm{C}$, the majority of the particles are not involved in the structures. The influence of magnetic field on the acoustic attenuation becomes small. The stability of such medium in a magnetic field is then better.

The anisotropy measurement can be interpreted using theories and from them to determine parameters of nanoparticle structures. At this type of measurement, the magnetic field is constant, and the angle $\phi$ between wave vector of acoustic wave $\boldsymbol{k}$ and the magnetic field $\boldsymbol{B}$ is changed. In Figure 5 there are presented results for the anisotropy of acoustic attenuation in $1 \%$ and $2 \%$ magnetic fluids based on TECHNOL. The solid lines represent the theoretical fit by Taketomi functions [7], [26] and symbols are experimental data. It can be seen that the changes of acoustics attenuation for $2 \%$ MF TECHNOL are higher as $1 \%$. Since the measurements show local maxima, we can say that some types of structures: oligomers, chains or clusters were created in the direction of magnetic field. Only in combination with a model, we can determine the shape and size of these structures. The observed results also indicate a strong effect of 
Table 1 Parameters describing 1\% and 2\% magnetic fluids based on the TECHNOL obtained from the fit of measured anisotropy data for two temperatures $\left(4 / 3 \boldsymbol{\eta}_{\mathrm{S}}+\boldsymbol{\eta}_{\mathrm{V}}\right.$ - bulk viscosity, a - radius of clusters, $\mathrm{N}$ the density of clusters and volume concentration of all clusters $\mathrm{V} \times \mathrm{N}$ )

\begin{tabular}{ccccc}
\hline MF & \multicolumn{3}{c}{$1 \%$} & \multicolumn{2}{c}{$2 \%$} \\
\hline Temperature & $20{ }^{\circ} \mathrm{C}$ & $30{ }^{\circ} \mathrm{C}$ & $20{ }^{\circ} \mathrm{C}$ & $30{ }^{\circ} \mathrm{C}$ \\
$4 / 3 \boldsymbol{\eta}_{\mathrm{S}}+\boldsymbol{\eta}_{\mathrm{V}}$ & 0.29 & 0.23 & 0.36 & 0.28 \\
$a[\mathrm{~nm}]$ & 50 & 24 & 138 & 37 \\
$10^{-17} \mathrm{~N}\left[\mathrm{~m}^{-3}\right]$ & 255 & 560 & 12 & 250 \\
$V \times N[\%]$ & 0.33 & 0.08 & 1.72 & 0.66 \\
\hline
\end{tabular}

temperature and concentration on the measured anisotropy of acoustic attenuation. The measurements at temperature $20{ }^{\circ} \mathrm{C}$ show the largest changes of acoustic attenuation because clusters around tithes nanometers in diameter were created. On the base of the calculated radius (Table 1 ) at the temperature $20^{\circ} \mathrm{C}$, we can say that the structures contain more as 6 nanoparticles for $1 \%$ and more as 15 nanoparticles for $2 \%$ MF. The changes of acoustic attenuation are smaller at higher temperatures. At temperature $30^{\circ} \mathrm{C}$ there are only 2-3 nanoparticles in the structure, so they can create only thin chains. Both smaller number of nanoparticles in clusters or chains and thus their shorter size induce the smaller influence of the direction of magnetic field with regards to the direction of acoustic wave propagation on the acoustic attenuation. We suppose that this effect is connected with smaller structures or chains due to Brownian motion.

Since nanoparticles are polarizable, the dielectric spectroscopy can be used to study their dielectric parameters. With the application of magnetic field, there are also changed dielectric parameters as complex permittivity and dissipation factor of MF. These changes are mainly caused by movement of electric charges in new structures of magnetic nanoparticles. The mechanisms of dielectric relaxations can be described from a measurement of the frequency dependence of dissipation factor. The low and high-frequency maximums should be clearly observed [29], [30]. On the basis of Maxwell-Wagner model, it was estimated that one maximum of $\tan \delta$ appears at hundreds of $\mathrm{MHz}$ frequency, which exhibits in many colloidal systems containing different dielectric constituents. The next low-frequency relaxation process, occurring in magnetic fluids is described by the Schwarz model [31], [32] Electric double layers are created on the particle surfaces as a result of electrostatic interactions between the particles and the space charge. Finally, the electrode polarization (EP) causes an anomalous increase in the complex dielectric permittivity in a lower frequency region by many orders of magnitude.

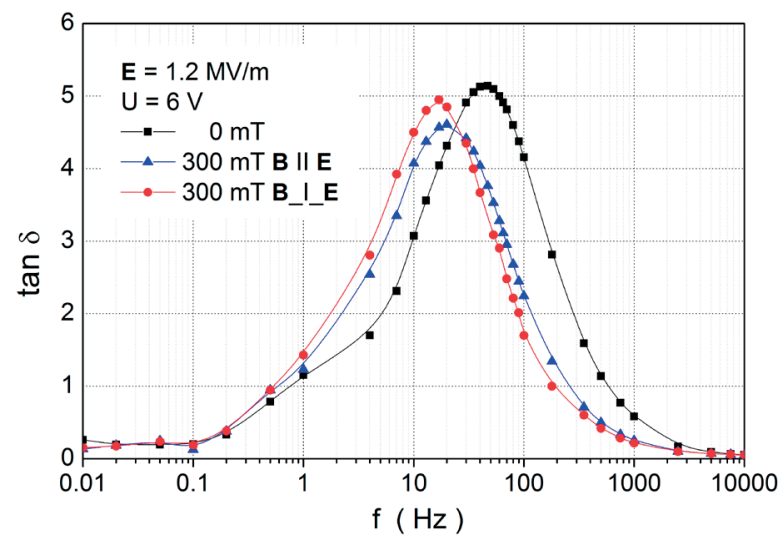

Figure 6 The effect of the external magnetic field and its orientation with respect to the electric field on the dissipation factor for $0.5 \%$ magnetic fluid based on MOGUL

In order to investigate the influence of the magnetic field on the electro-kinetics within the magnetic fluid, the whole system was placed inside an electromagnet. One of the measurements for the frequency dependence of dissipation factor of $\mathrm{MF}$ based on transformer oil MOGUL measured in the range from $0.01 \mathrm{~Hz}$ to $10 \mathrm{kHz}$ is depicted in Figure 6. The interesting changes are significant for frequencies lower than $1 \mathrm{kHz}$. There is clearly observed a low-frequency relaxation maximum, the position of which also depends on various external factors. In this figure, the magneto-dielectric effect of studied MF is presented. When the external magnetic field was applied the whole development of the dissipation factor shifted to the left side. In general, we can say that at the parallel orientation of fields the eigenfrequency is smaller than for perpendicular orientation and without the application of magnetic field. The change of dissipation factor with the application of magnetic field results from a rearrangement of nanoparticles to new structures, as oligomers or chains. These agglomerations have electric dipoles so generate higher depolarization field, which further reduces the total intensity of electric field. Consequently, the relaxation maximum shifts towards lower frequencies with the application of magnetic field. For the perpendicular orientation of the electric field to the magnetic field, the Lorentz force has a maximum effect on the drift of the nanoparticles in the direction of the electric field. This force causes the motion of nanoparticles in a spiral, which causes longer tracks of the nanoparticles in the system and it is connected with the increase of dissipation factor. The position of the local maximum of $\tan \delta$ for parallel orientation of fields is at the frequency of $20 \mathrm{~Hz}$ and for perpendicular $17 \mathrm{~Hz}$. From the fit of the measured complex permittivity of ferrofluid using the Havriliak-Negani relationship resulted that the DC conductivity of the ferrofluid slightly increases within the magnetic field [31]. This could be the result of new structures from nanoparticles which have higher charge; in general, they increase the conductivity. 


\section{Conclusion}

The applications of magnetic fluids are very useful for many technological, biological and medical purposes, and they can also help in materials science and engineering research. For the correct understanding of processes in a magnetic fluid at the application of a magnetic field, it is important to have various diagnostic methods. One of them is the acoustic spectroscopy, which is a very useful tool for investigation of magnetic fluid subjected to an influence of a magnetic field. The acoustic attenuation depends on temperature, type, and development of a magnetic field. The study of the dielectric properties of a magnetic fluid in the magnetic field is the next method. Particle agglomeration within a magnetic fluid and the magneto-dielectric effect can be distinguished by the dielectric spectroscopy. Combinations of these methods bring more complex view on agglomeration and electric properties of a magnetic fluid.

\section{Acknowledgment}

This work was supported by KEGA 012TU Z-4/2017.

\section{References}

[1] TKACOVA, Z., LAVICKY, T.: Introduction to Nanoscience and Nanotechnologies for Teachers (in Slovak). Metodickopedagogicke centrum v Bratislave, 2014.

[2] CHARLES, S. W., POPPLEWELL, J.: Ferromagnetic Material. WOHFARTH, E. P. (Ed.). North-Holland Publishing Company, Vol. 2, Amsterdam, 1980.

[3] SCHERER, C., FIGUEIREDO NETO, A. M.: Ferrofluids: Properties and Applications. Brazilian Journal of Physics, 35(3A), 718$727,2005$.

[4] POLUNIN, V.: Acoustics of Nanodispersed Magnetic Fluids. CRC Press Taylor, 2015.

[5] BUHRMAN, R. A., GRANQVIST, C. G.: Log-Normal Size Distributions from Magnetization Measurements on Small Superconducting AI Particles. Journal of Applied Physics, 47, 2220, 1976.

[6] JOZEFCZAK, J., HORNOWSKI, T., ZAVISOVA, V., SKUMIEL, A., KUBOVCIKOVA, M., TIMKO, M.: Acoustic Wave in a Suspension of Magnetic Nanoparticle with Sodium Oleate Coating. Journal of Nanoparticle Research, 16, 2271, 2014.

[7] TAKETOMI, S.: The Anisotropy of the Sound Attenuation in Magnetic Fluid under an External Magnetic Field. Journal of the Physical Society of Japan, 55, 838-844, 1986.

[8] AHUJA, A., HENDEE, W.: Effect of Particle Shape and Orientation on Propagation of Sound in Suspensions. The Journal of the Acoustical Society of America, 64, 1074-1080, 1978.

[9] SHLIOMIS, M., MOND, M., MOROZOV, K.: Ultrasound Attenuation in Ferrofluids. Physics Review Letter, 101, 074505, 2008.

[10] SATOH, A.: Three-Dimensional Monte Carlo Simulations of Internal Aggregate Structures in a Colloidal Dispersion. Journal of Colloid and Interface Science, 318, 68-81, 2008.

[11] VINOGRADOV, A. N.: Application of Acoustic Spectroscopy to Investigation of Microinhomogeneous Media. Colloid Journal, 65, 539-544, 2003.

[12] SOKOLOV, V. V.: Wave Propagation in Magnetic Nanofluids (A Review). Acoustical Physics, 6, 972-988, 2010.

[13] PLEINER, H., BRAND, H. R.: The Anisotrophy of the Macroscopis Equations for Ferrofluids. Journal of Magnetism and Magnetic Materials, 85, 125-159, 1990.

[14] SHLIOMIS, M. I.: Magnetic Fluids. Soviet Uspekhi, 17-34, 1974.

[15] ODENBACH, S.: Ferroluids. Magnetically Controllable Fluids and their Applications. Springer-Verlag, 2002.

[16] ODENBACH, S.: Ferofluids. Handbook of Magnetic Materials, 16, chap. 3, 127-208, 2006.

[17] SHI, D.: NanoScience in Biomedicine. Springer, 2009.

[18] YI, D. K., PAPAEFTHYMIOU, G. C.: Nanobiomaterials Development and Application (eBook - PDF). CRC Press, 2013.

[19] GENNES, P. G., PROST, J.: The Physics of Liquid Crystals. Clarendon Press, Oxford 1993.

[20] VEVERICIK, M., BURY, P., KOPCANSKY, P., TIMKO, M., MITROOVA, Z.: Effect of Carbon Nanotubes on Liquid Crystal Behavior in Electric and Magnetic Fields Studied by SAW. Procedia Engineering, 192, 935 - 940, 2017.

[21] FIGUEIREDO NETO, A. M., SABA, M. M. F.: Determination of the Minimum Concentration of Ferrofluid Required to Orient Nematic Liquid Crystals. Physical Review A 34, 3483-3485, 1986.

[22] BACRI, J. C., FIGUEIREDO NETO, A. M.: Dynamics of Lyotropic Ferronematic Liquid Crystals Submitted to Magnetic Fields. Physical Review E, 50, 3860, 1994. 
[23] GOMEZ, S. L., CUPPO, F. L. S., FIGUEIREDO NETO, A. M.: Nonlinear Optical Properties of Liquid Crystals Probed by Z-Scan Technique. Brazilian Journal of Physics, 33, 813-820, 2003.

[24] KHOO, I. C.: Liquid Crystals: Physical Properties and Nonlinear Optical Phenomena. Wiley, New York, 1987.

[25] KUDELCIK, J., BURY, P., DRGA, J., KOPCANSKY, P., ZAVISOVA, V., TIMKO, M.: Structure of Transformer Oil-Based Magnetic Fluids Studied Using Acoustic Spectroscopy. Journal of Magnetism and Magnetic Material, 326(1), 75-80, 2013.

[26] KUDELCIK, J., BURY, P., KOPCANSKY, P., TIMKO, M.: Structure of Nanoparticles in Transformeroil-Based Magnetic Fluids, Anisotropy of Acoustic Attenuation. Journal of Magnetism and Magnetic Material, 388, 28-34, 2015.

[27] JOZEFCZAK, J., LABOWSKI, M., SKUMIEL, A.: Hysteresis of Changes of Ultrasonic Wave Absorption Coefficient in a Magnetic Fluid Caused by the Magnetic Field. Journal of Magnetism and Magnetic Materials, 252, 356-359, 2002.

[28] KUDELCIK, J., HARDON, S., BURY, P., TIMKO, M., KOPCANSKY, P.: Study of Structural Changes of Water-Based MagneticFluid by Acoustic Spectroscopy. Acta Physica Polonica A, 131(4), 919-921, 2017.

[29] KUDELCIK, J., HARDON, S., VARACKA, L.: Measurement of Complex Permittivity of Oil-Based Ferrofluid in Magnetic Field. Acta Physica Polonica A, 131(4), 931-933, 2017.

[30] SPANOUDAKI, A., PELSTER, R.: Frequency Dependence of Dielectric Anisotropy in Ferrofluids, Journal of Magnetism and Magnetic Material, 252, 71-73, 2002.

[31] RAJNAK, M., KURIMSKY, J., DOLNIK, B., MARTON, K., TOMCO, L., TACUESCU, A., and et al: Dielectric Response of Transformer Oil Based Ferrofluid in Low Frequency Range, Journal of Applied Physics, 114, 034313, 2013.

[32] LIU, J., ZHOU, L., WU, G., ZHAO, Y.: Dielectric Frequency Response of Oil-Paper Composite Insulation Modified by Nanoparticles. IEEE Transactions on Dielectrics and Electrical Insulation, 19, 510-520, 2012. 